\title{
The Performance Comparison among Common Struts of Heavy-duty Gas Turbine
}

\author{
Jian $\mathrm{Li}^{1, \mathrm{a}}$, Jian Zhou ${ }^{1, \mathrm{~b}}$, Guohui $\mathrm{Xu}{ }^{1, \mathrm{~b}}$, Lie $\mathrm{Yu}^{1, \mathrm{~b}}$ and Xinping $\mathrm{Yan}^{2, \mathrm{c}}$ \\ ${ }^{1}$ Xi'an Jiaotong University, Xi'an 710049, Shaanxi, China; \\ ${ }^{2}$ Wuhan University of Technology, Wuhan 430063, hubei, China. \\ ajianli0128@163.com, bjzhou@mail.xjtu.edu.cn, cxpyan@whut.edu.cn
}

Keywords: Radial struts, Tangential struts, Stress, Stiffness.

\begin{abstract}
In order to study the performance differences among struts of a heavy-duty gas turbine, three-dimension finite-element models of several struts were established by Pro/Engineer. Firstly, the stresses of three struts were obtained only considering the rotor gravity. The magnitudes and distributions of stress and influences of the width and thickness for support plates on stiffness are studied. The results show that radial struts 2 are superior to radial struts 1 and tangential struts is superior to radial struts. The sensitivity of stiffness to the width of support plates is greater than the thickness. Then, the distribution of temperature was obtained by the thermo-fluid-solid coupled method in CFD software to calculate the thermal stress of radial and tangential struts. The results show that although the magnitudes and distributions of temperature are similar, the thermal stress of tangential struts is smaller than that of radial struts.
\end{abstract}

\section{Introduction}

The gas turbine is widely used in aviation, power generation, metallurgy, chemical industry, ship building, energy and power engineering as power plant. In recent years, the manufacturing technology of gas turbine has become one of the important symbols to measure the level of science and technology, defense and comprehensive strength of a country, which is known as the "Pearl" in the manufacturing industry [1].When heavy-duty gas turbine is working, high temperature gas and cooling air will go through struts in the hot end which makes the working conditions of struts to be severe. The struts not only bear the rotor gravity, but also the impact of high temperature and pressure. High temperature gas can result in the thermal deformation and thermal stress of struts, so in order to decrease the influence of thermal deformation on the shafting center elevation, how to select the proper form of struts is very important for most gas turbine manufacturers to ensure safe operation of gas turbine [2]. The studies on the form of gas turbine struts in foreign countries started early. Japan Mitsubishi developed and manufactured a tangential strut with six tangential support plates [Y. Fukuizumi et. al, Journal of Engineering for Gas Turbines and Power, 2005, Vol. 127, pp.369-374] [3]. China also introduced this technology, which is being applied in DongFang Steam Turbine plant. Other types of struts have also been developed and patented, such as the American [United States Patent, Pn.5483792, Jan.16, 1996] [4], the Europe [European Patent, EP 1775428A2, 8.04.2007] [5], the Germany [Deutsches Patentamt, DE 4329623A1, 9.04.1995] [6] etc.

In this paper, several common struts, as shown in Fig. 1 will be studied. Firstly, only considering the rotor gravity, the stress of support plates and influence of the width and height for support plates on stiffness are studied. Then, the temperature distributions of radial and tangential struts were obtained through the thermo-fluid-solid coupled method in CFD software to calculate the thermal stress of them. Based on the computed results, the analysis and comparison of several common struts are conducted. 


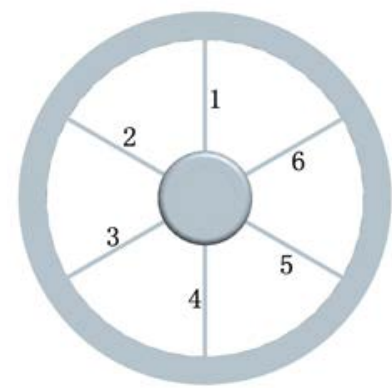

(a)Radial struts 1

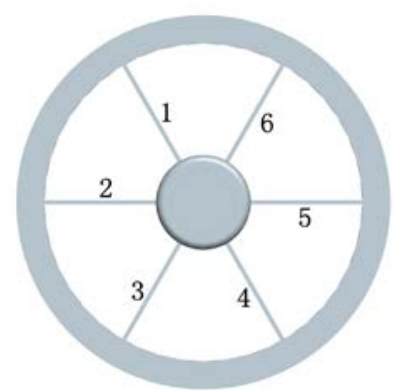

(b) Radial struts 2

Fig. 1 The Structure of Common Struts.

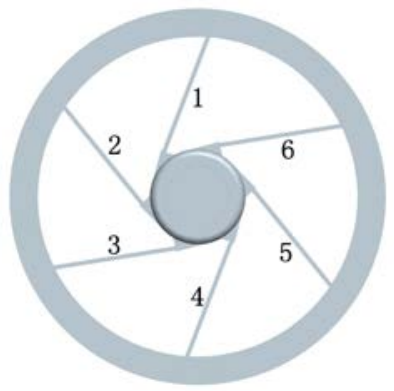

(c) Tangential struts

\section{Basic Theory for Resolving Temperature Field and Stress Field}

Calculation of magnitude and distribution of struts stress in this paper is related to solid total deformation problem under the action of external load. It can be represented by the principle of virtual work, and the equation is as follows:

$$
\int_{\Omega} D_{i j k l} \varepsilon_{k l} \delta \varepsilon_{i j} \mathrm{~d} \Omega-\left(\int_{S_{p}} \bar{p}_{i} \delta u_{i} \mathrm{~d} A+\int_{\Omega} \bar{b} \delta u_{i} \mathrm{~d} \Omega\right)=0
$$

In the equation, the first item on the left represents internal stress produced by the external load, the second item represents pressure and the third one represents external force.

The thermo-fluid-solid coupled method in this paper mainly involves the fluid flow and heat transfer process between fluid and solid. It mainly includes three physical problems: (1) the turbulent flow of fluid which goes through struts in the hot end; (2) the convective heat transfer between fluid and solid wall of struts; (3) the heat conduction in the struts. In order to transform the above three physical problems into the mathematical problems, it should be described by three mathematical models which include the fluid flow, convective heat transfer and heat conduction of solid[7]. The specific equations are as follows.

The continuity equation:

$$
\frac{\partial \rho}{\partial t}+\frac{\partial}{\partial x_{i}}\left(\rho u_{i}\right)=0
$$

The momentum equation:

$$
\frac{\partial}{\partial x_{j}}\left(\rho u_{i} u_{j}\right)=-\frac{\partial p}{\partial x_{i}}+\left[u\left(\frac{\partial u_{i}}{\partial x_{j}}+\frac{\partial u_{j}}{\partial x_{i}}\right)-\frac{2}{3} \delta_{i j} \frac{\partial u_{i}}{\partial x_{j}}\right]+\frac{\partial}{\partial x_{j}}\left(-\rho \vec{u}_{i}^{\prime} \vec{u}_{j}^{\prime}\right) .
$$

Transport equation of the turbulent kinetic energy $k$ :

$$
\frac{\partial}{\partial t}(\rho k)+\frac{\partial}{\partial x_{i}}\left(\rho k u_{i}\right)=\frac{\partial}{\partial x_{j}}\left(\alpha_{k} \mu_{\mathrm{eff}} \frac{\partial k}{\partial x_{j}}\right)+G_{k}+G_{b}-\rho \varepsilon-Y_{M} .
$$

Transport equation of the turbulent dissipation rate $\varepsilon$ :

$$
\frac{\partial}{\partial t}(\rho \varepsilon)+\frac{\partial}{\partial x_{i}}\left(\rho \varepsilon u_{i}\right)=\frac{\partial}{\partial x_{j}}\left(\alpha_{k} \mu_{\text {eff }} \frac{\partial \varepsilon}{\partial x_{j}}\right)-C_{2 \varepsilon} \rho \frac{\varepsilon^{2}}{K}+G_{1 \varepsilon} \frac{\varepsilon}{k}\left(G_{k}+C_{3 \varepsilon} G_{b}\right) \text {. }
$$

where $i, j=1,2,3 ; u=$ velocity of medium; $p=$ pressure of medium; $\mu$ = viscosity of medium; $\delta_{i j}$ = Kronecker operator; $K=$ turbulence intensity of fluid; $\varepsilon=$ turbulent dissipation rate of fluid; $\mu_{\text {eff }}=$ turbulent viscosity; $Y_{M}=$ turbulent expansion dissipation; $G_{k}=$ turbulent kinetic energy related to the average velocity gradient; $G_{b}=$ turbulent kinetic energy related to buoyancy; $C_{\mu}=0.09 ; C_{1 \varepsilon}=1.44$; $C_{2 \varepsilon}=1.92 ; \sigma_{k}=1 ; \sigma_{\varepsilon}=1.3$.

The principle of conservation of energy is used to describe the process of convective heat transfer as equation (6): 


$$
\rho \frac{\mathrm{D}}{\mathrm{D} t}\left(h+\frac{1}{2} u^{2}\right)-\frac{\partial p}{\partial t}=0
$$

where $h=$ enthalpy of flow medium.

Also, the heat conduction in the solid wall of struts should meet the principle of conservation of energy. Because the effect of pressure on heat transfer of solid is very small, assuming that the process of heat conduction of solid is steady, the process of heat conduction of solid can be simplified as the formula (7):

$$
\nabla \cdot(k \nabla T)=0
$$

The heat transfer between fluid and solid wall should also meet the principle of conservation of energy. That is, on the contact surface of fluid and solid, the total heat absorbed by solid should be the same as that released by fluid. So the process of energy transfer on the contact surface of fluid and solid can be represented with formula (8):

$$
\lambda\left(\frac{\partial T_{W}}{\partial X}\right)=\alpha\left(T_{W}-T_{f}\right) .
$$

where $\lambda=$ solid thermal conductivity; $\alpha=$ convective heat transfer coefficient; $T_{w}=$ solid wall temperature; $T_{f=}$ fluid temperature.

The temperature of structures rises rapidly considering the action of high-temperature gas. According to the basic theory of finite element [8], finite element equation of strength of struts can be obtained by the minimum energy principle.

$$
k \delta=W \text {. }
$$

where $K=$ the stiffness matrix of the structure; $\delta=$ displacement vector; $W=$ resultant of forces which fluid on solid surface.

During calculating the stiffness matrix by using finite element method, when the base is discrete, the above formula also can be written as [9]:

$$
\sum_{e} \int_{v^{e}} B^{T} \sigma \mathrm{d} v^{e}=\sum_{e} F^{e}+\sum_{e} P^{e}+\sum_{e} R^{e}
$$

where $e=$ calculation unit; $v_{e}=$ calculation cell cube; $\sigma=$ stress vector of one point in unit; $B=$ Strain matrix of unit; $F=$ body force load vector of unit; $P=$ surface force load vector of unit; $R=$ concentrated force load vector of unit.

The temperature distribution can be obtained by the thermo-fluid-solid coupled method for engine base, diffuser cones, hot and cold fluid channel element in CFD software, and then the deformation and thermal stress of radial and tangential struts can be calculated.

\section{Performance Comparisons of Several Struts Considering Rotor Gravity}

Deformation and stress can be produced because of the rotor gravity of heavy-duty gas turbine. Only considering the rotor gravity, according to the different distribution forms of support plates in radial struts, the magnitudes and distributions of stress for three struts are obtained by ANSYS software. Meanwhile, the influences of the width and height for support plates on stiffness are studied. The rotor gravity is about $50 \mathrm{kN}$. Models of three struts are shown in Fig. 1. 


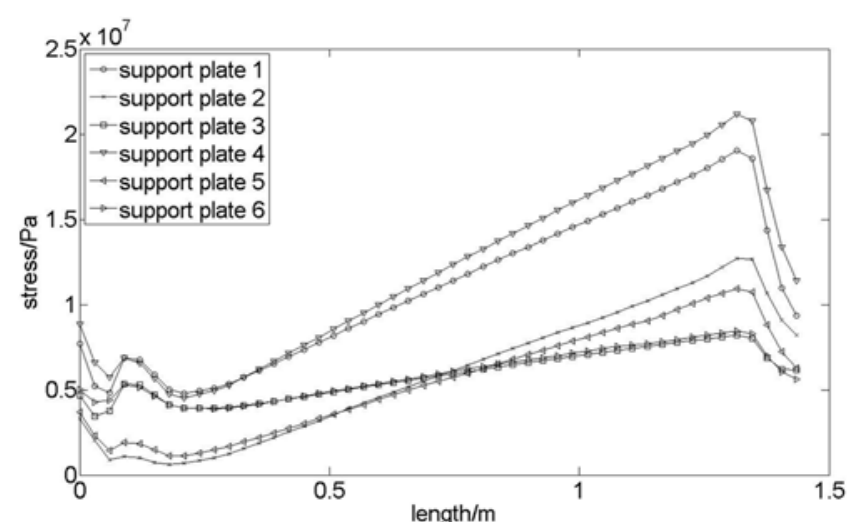

(a) The stress of support plates for radial struts 1 2

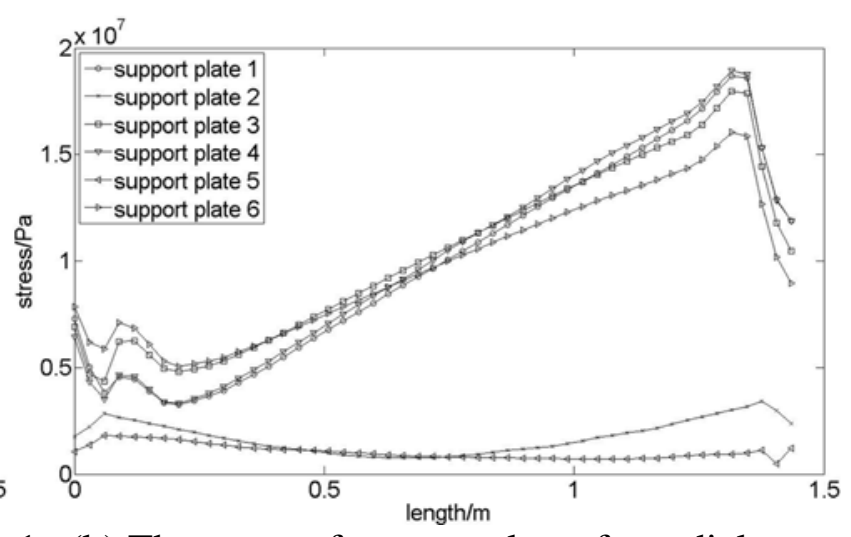

(b) The stress of support plates for radial struts

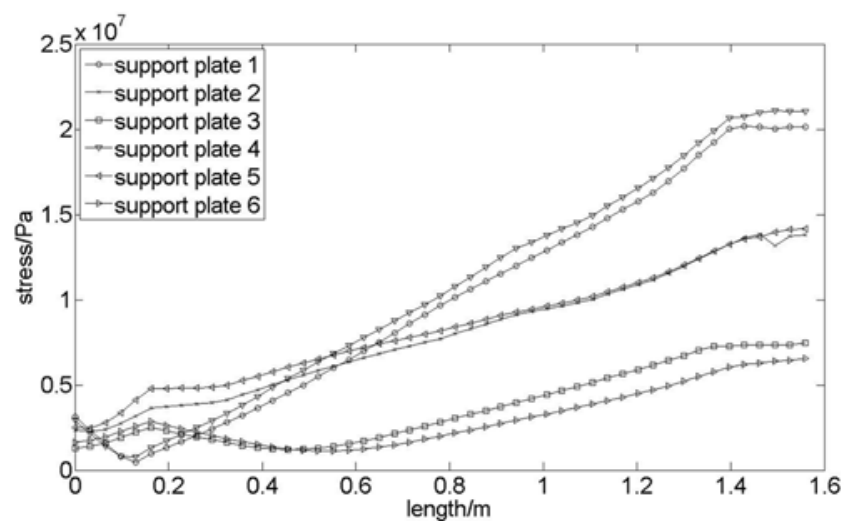

(c) The stress of support plates for tangential struts

Fig. 2 Stress-length curves of support plates for three struts.

Fig. 2 shows the stress-length curves of six support plates for three struts. For radial struts 1 , the rotor gravity is mainly supported by support plate 1 and 4 . The tensile stress occurs in the support plate 1 and the compressive stress occurs in the support plate 4 . For radial struts 2 , the rotor gravity is mainly supported by support plate 1, 3, 4 and 6. They bear the bending stress except the tensile and compressive stress. The stresses of support plates for radial struts 2 are smaller than those for radial struts 1 . So, radial struts 2 are superior to radial struts 1 considering the rotor gravity. For tangential struts, the stresses of support plates are symmetrical. So it can be concluded that the stress homogenization of tangential struts is superior to radial struts.

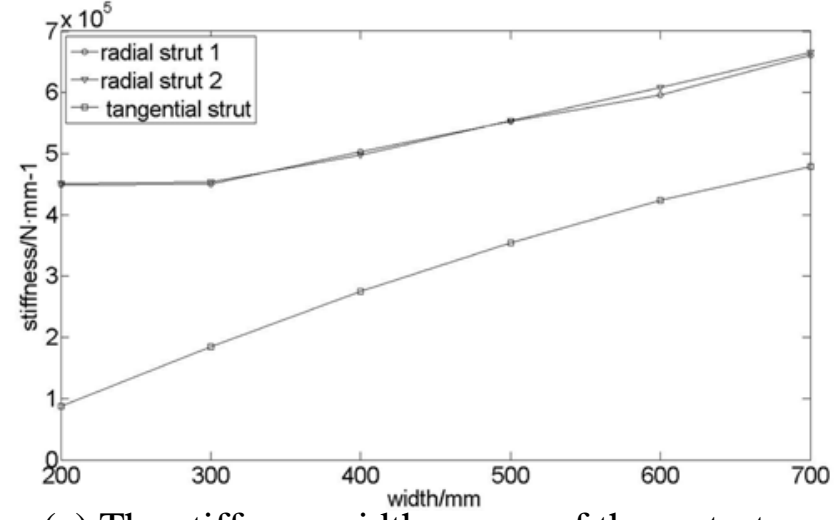

(a) The stiffness-width curves of three struts

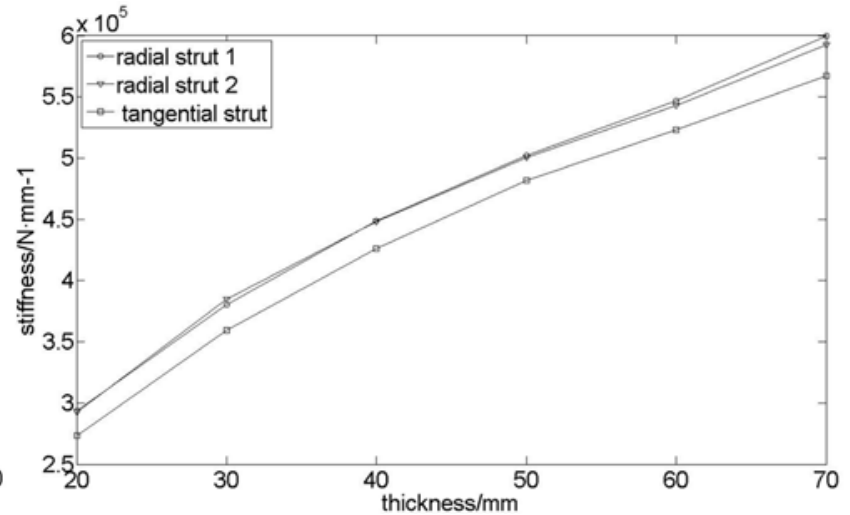

(b) The stiffness- thickness curves of three

Fig. 3 Stiffness-width and Stiffness-thickness Curves of Three Struts.

Fig. 3 shows the stiffness-width and stiffness-thickness curves of three struts. The stiffness increases with the growing of width and height for support plates. It can be seen that stiffness of tangential struts is smaller than that of radial struts and the stiffness of radial struts 1 and 2 are similar in the same width and thickness of support plates. The results also show that the sensitivity of stiffness to the width of support plates is greater than thickness. 


\section{Performance Comparisons of Radial and Tangential Struts Under High Temperature Gas}

When heavy-duty gas turbine is working, high temperature gas and cooling air go through struts in the hot end, so working conditions of struts are extremely abominable due to bearing the impacts of high temperature, pressure and speed. The thermal stress of struts resulted from the temperature field can affect the stability and safety of shafting operation. The magnitude and distribution of temperature and thermal stress of radial and tangential struts were analyzed by workbench considering high temperature gas and cooling gas.

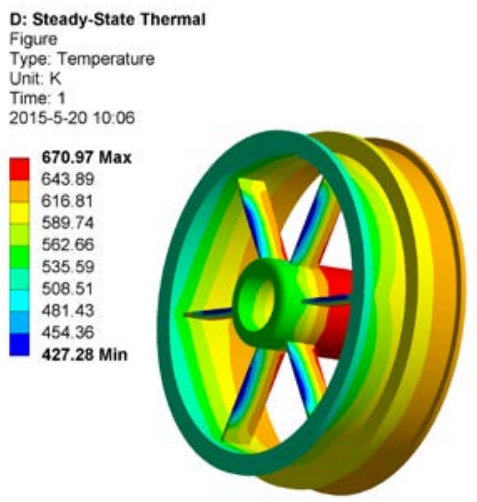

(a) The temperature field of radial struts

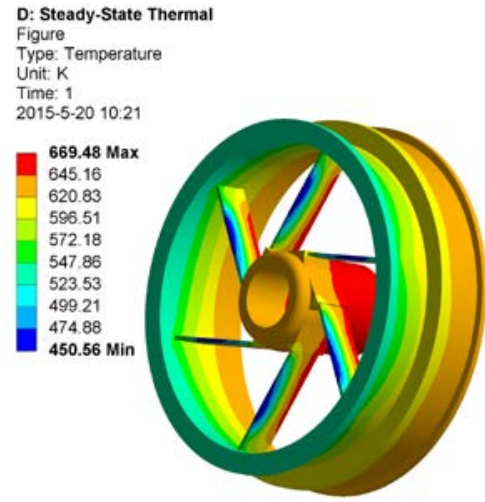

(b) The temperature field of tangential struts

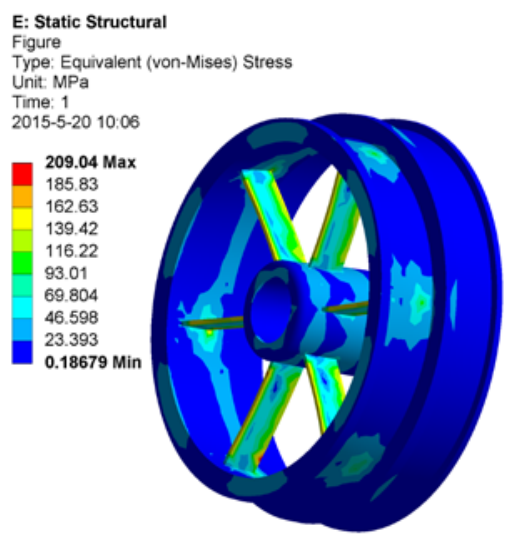

(a) Thermal stress field of radial struts

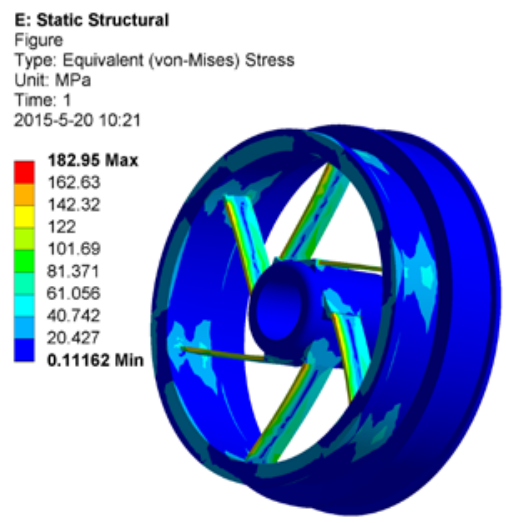

(b) Thermal stress field of tangential struts

Fig. 5 Thermal Stress Distributions of Struts

Temperature distributions of radial and tangential struts are shown in the Figure 4a,b, and thermal stress distributions of radial and tangential struts are shown in the Figure 5a,b. It can be seen from Figure 4 and 5 that the magnitude and distribution of temperature for radial and tangential struts are similar, but the magnitude of thermal stress has great difference when high temperature gas and cooling gas go through them. The temperature in the front of struts is smaller than that in the back and the temperature of struts is symmetric. The lowest temperature and the highest temperature of two struts are about $440 \mathrm{~K}$ and $670 \mathrm{~K}$ respectively. The thermal stress of struts is also symmetric. The stress of support plates is larger than other parts for radial and tangential struts. The maximum stress occurs in the joints of support plates and shell and bearing pedestal. The maximum thermal stresses of radial and tangential struts are $210 \mathrm{MPa}$ and $182 \mathrm{MPa}$ respectively. It can be seen that thermal stress of tangential struts is lower than that of radial struts. Therefore, tangential struts are superior to radial struts in the same temperature field.

\section{Summary}

(1) Only considering the rotor gravity, the stresses of support plates for radial struts 2 are smaller than those for radial struts 2 and the stress homogenization of tangential struts is superior to that of radial struts. So it can be concluded that radial struts 2 are superior to radial struts and tangential struts are superior to radial struts considering the rotor gravity. 
(2) Stiffness of tangential struts is smaller than that of radial struts and the stiffness of radial struts 1 and 2 are similar in the same width and thickness of support plates. When the width and height of support plates are changed, in can be seen that the sensitivity of stiffness to the width of support plates is greater than thickness. So the width of support plates should be given priority to be concerned in the matching of stiffness for struts.

(3) Under high temperature gas and cooling gas, the stress of support plates is larger than other parts for radial and tangential struts. Stress concentration which is prone to fatigue failure occurs in the joints of support plates and shell and bearing pedestal. Although the magnitude and distribution of temperature for radial and tangential struts is similar, the maximum stress of tangential struts is less than that of radial struts. Therefore, tangential struts are better than radial struts for reducing thermal stress.

\section{Acknowledgments}

The work described in this paper is supported by the National Program on Key Basic Research Projects of China (Grant No.2013CB035705), National Natural Science Foundation of China (Grant No. 51175411, 11102144, 51375366, 51139005), Shanxi Provincial Natural Science Foundation of China (2011JM1005).

\section{References}

[1] J.Q. Han, Gas turbine heat transfer and cooling technology. Xian: Xi'an Jiaotong University. (2005)13-15.

[2] Y. FUKUIZUMI, J. MASADA, V. KALLIANPUR, Y. LWASAKI, Application of "H gas turbine" design technology to increase thermal efficiency to increase thermal efficiency and output capability of the Mitsubishi M701G2 gas turbine. Trans. ASME, journal of engineering for gas turbines and power. 127(2005) 369-374.

[3] B.L. Newkirk, H.D. Taylor, Shaft whipping due to oil action in journal bearing, General Electric Review.(1925).

[4] K. Huseyin, Vibration and Stability of Multiple Parameter Systems, Noordhoff International Publishing. (1978).

[5] G. Adiletta, A.R. Guido, C. Rossi, Chaotic motions of a rigid rotor in short journal bearing. Nonlinear Dynamics.(1996)0:251-269.

[6] Y.N. Robotnov, Creep rupture process. Ptoc.12th Int. Cong. Appl. Mesh, IUTAM. (1968) 567-578.

[7] W.S. TAO, Numerical heat transfer. Xian: Xi'an Jiaotong University Press. (2004) 347-362.

[8] W.L. Liao, B. Xu, P. Lu, X.Q. Luo, Influence of runner blade deformation on fluid field of francis turbine under part load. Chinese Journal of Mechanical Engineering. (2006)55-59.

[9] C. TREVINO, A. ESPINOZA, F. MENDEZ. Steady-state analysis of the conjugate heat transfer between forced counter flowing streams, ASME J. Thermophys. Heat Transfer 10(1996)476-483. 